Journal of Mathematics and Statistics 6 (1): 56-59, 2010

ISSN 1549-3644

(C) 2010 Science Publications

\title{
Split-Quaternionic Representation of the Moving Frame for Timelike Surfaces in 3-Dimensional Minkowski Spacetime
}

\author{
Paul Bracken \\ Department of Mathematics, University of Texas, Edinburg, TX 78541-2999
}

\begin{abstract}
The moving frame and associated Gauss-Codazzi equations for surfaces in Minkowski three space are introduced. A split-quaternionic representation is used to identify the Gauss-Weingarten equations with a Lax pair representation. This Lax pair representaion is calculated and given explicitly.
\end{abstract}

Key words: Split-quaternions, Lax pair, Gauss-Codazzi equation

\section{INTRODUCTION}

The study of surfaces in higher-dimensional spaces and in non-Euclidean spaces has been an active area of study recently due to the variety of applications of surfaces to the areas of integrable systems and mathematical physics (Bracken and Grundland, 1999; Bracken et al., 1999). Recently, the Gauss-Codazzi equations for surfaces in Euclidean three-space were established from a three by three matrix representation and a quaternionic representation was introduced for the moving frame of the conformally parametrized surface (Konopelchenko and Taimanov, 1996). This work is extended here in what is intended to be a companion paper (Konopelchenko and Taimanov, 1996; Bracken, 2004). Here a hyperbolic version of what was done in (Bracken, 2004) is presented. For this other case, it will be shown that a Lax pair can be derived for the Gauss-Codazzi equations by using a representation of the split-quaternions (Bracken and Hayes, 2002). This algebraic structure will be defined and some of their relevant properties will be given. It will be shown how the frame equations can be written down using a matrix representation for the splitquaternions (Inoguchi, 1998; 1999).

To begin to introduce Minkowski three-space, let $\mathrm{E}_{1}^{3}=\left\langle\mathbb{R}^{3},\langle.,\rangle.\right\rangle \quad$ denote Minkowski three-space (Dorfmeister et al., 2002; Inoguchi, 1998) under the natural metric $\langle.,$.$\rangle which can be expressed in terms of$ the natural coordinate system as $\langle.,\rangle=.-\mathrm{d} \xi_{1}^{2}+\mathrm{d} \xi_{2}^{2}+\mathrm{d} \xi_{3}^{2}$. Let $\mathrm{M}$ be a connected two-manifold and $\varphi: M \rightarrow E_{1}^{3}$ an immersion. The immersion $\varphi$ is said to be timelike if the induced metric I on $\mathrm{M}$ is Lorentzian (Weinstein, 1996).

Let us assume $\mathrm{M}$ is an orientable time-like surface in $E_{1}^{3}$ immersed by $\varphi$. The Lorentzian metric of a timelike surface $M$ determines a conformal structure with $\varphi$ as a conformal immersion. On a timelike surface $\mathrm{M}$, there exists a local coordinate system (x, y) such that:

$I=e^{\omega}\left(-d x^{2}+d y^{2}\right)$

This is referred to as a Lorentz isothermal coordinate system. Let $(\mathrm{u}, \mathrm{v})$ be the null coordinate system obtained from $(\mathrm{x}, \mathrm{y})$ by a rotation, such that:

$\mathrm{u}=\mathrm{x}+\mathrm{y} ; \mathrm{v}=-\mathrm{x}+\mathrm{y}$

The partial derivatives of $\varphi$ satisfy:

$\left\langle\varphi_{\mathrm{u}}, \varphi_{\mathrm{u}}\right\rangle=\left\langle\varphi_{\mathrm{v}}, \varphi_{\mathrm{v}}\right\rangle=0,\left\langle\varphi_{\mathrm{u}} ; \varphi_{\mathrm{v}}\right\rangle=\frac{1}{2} \mathrm{e}^{\omega}$

If $\mathrm{H}$ is the mean curvature function, then $\mathrm{H}=2 \mathrm{e}^{-\omega}\left\langle\varphi_{\mathrm{uv}}, \mathrm{N}\right\rangle$ and $\mathrm{Q}$ and $\mathrm{R}$ will be given as $\mathrm{Q}=\left\langle\varphi_{\mathrm{uv}}\right.$, $\mathrm{N}\rangle$ and $\mathrm{R}=\left\langle\varphi_{\mathrm{uv}}, \mathrm{N}\right\rangle$. The first and second fundamental forms are given with respect to the Lorentz isothermal coordinate system as follows:

$$
\begin{aligned}
& M_{I}=e^{\omega}\left(\begin{array}{cc}
-1 & 0 \\
0 & 1
\end{array}\right) \\
& M_{I I}=-\langle d \varphi, d N\rangle=\left(\begin{array}{cc}
Q+R+H e^{\omega} & Q-R \\
Q-R & Q+R+H e^{\omega}
\end{array}\right)
\end{aligned}
$$

From these, the product $\mathrm{M}_{\mathrm{II}} \cdot \mathrm{M}_{\mathrm{I}}^{-1}$ can be calculated:

$$
\mathrm{M}_{\mathrm{II}} \cdot \mathrm{M}_{\mathrm{I}}^{-1}=\left(\begin{array}{cc}
\mathrm{Q}+\mathrm{R}-\mathrm{He}^{\omega} & \mathrm{Q}-\mathrm{R} \\
\mathrm{R}-\mathrm{Q} & \mathrm{Q}+\mathrm{R}+\mathrm{He}^{\omega}
\end{array}\right) \mathrm{e}^{-\omega}
$$

The matrix in (5) has the characteristic polynomial: 


\section{$t^{2}-2 H t+H^{2}-4 Q R e^{-2 \omega}=0$}

The roots of (6) give the principal curvatures of the surface, namely $\kappa_{1,2}=\mathrm{H} \pm 2 \sqrt{\mathrm{QR}} \mathrm{e}^{-\omega}$.

The average of these two just yield $\mathrm{H}$, the mean curvature and the product gives the Gaussian curvature:

$\mathrm{K}=\mathrm{H}^{2}-4 \mathrm{QRe} \mathrm{e}^{-2 \omega}$

A moving frame is defined by $\sigma=\left(\varphi_{\mathrm{u}}, \varphi_{\mathrm{v}}, \mathrm{N}\right)^{\mathrm{T}}$, which is required to satisfy the following GaussWeingarten equations:

$\sigma_{\mathrm{u}}=\mathrm{U} \sigma, \quad \sigma_{\mathrm{v}}=\mathrm{V} \sigma$

where, $\mathrm{U}$ and $\mathrm{V}$ are defined to be the matrices:

$$
\mathrm{U}=\left(\begin{array}{ccc}
\omega_{\mathrm{u}} & 0 & \mathrm{Q} \\
0 & 0 & \frac{1}{2} \mathrm{He}^{\omega} \\
-\mathrm{H} & -2 \mathrm{Qe}^{\omega} & 0
\end{array}\right), \mathrm{V}=\left(\begin{array}{ccc}
0 & 0 & \frac{1}{2} \mathrm{He}^{\omega} \\
0 & \omega_{\mathrm{u}} & \mathrm{R} \\
-2 \mathrm{Re}^{-\omega} & -\mathrm{H} & 0
\end{array}\right)
$$

It is required that system (8) satisfy the compatibility condition $\sigma_{\mathrm{uv}}=\sigma_{\mathrm{vu}}$. This in turn implies that $\mathrm{U}$ and $\mathrm{V}$ in (9) satisfy the condition:

$\mathrm{U}-\mathrm{V}+\mathrm{UV}-\mathrm{VU}=0$

Taking the two matrices in (9) and substituting them into (10), the left-hand side of (10) takes the following form:

$$
\left(\begin{array}{cc}
\omega_{\mathrm{uv}}+\frac{1}{2} \mathrm{H}^{2} \mathrm{e}^{\omega}-2 \mathrm{RQe}^{-\omega} & 0 \\
0 & -\omega_{\mathrm{uv}}-\frac{1}{2} \mathrm{H}^{2} \mathrm{e}^{\omega}+2 \mathrm{RQ}^{-\omega} \\
-\mathrm{H}_{\mathrm{v}}+2 \mathrm{R}_{\mathrm{u}} \mathrm{e}^{-\omega} & \mathrm{H}_{\mathrm{u}}+2 \mathrm{Q}_{\mathrm{v}} \mathrm{e}^{-\omega} \\
& \mathrm{Q}_{\mathrm{v}}+\frac{1}{2} \mathrm{H}_{\mathrm{u}} \mathrm{e}^{\omega} \\
-\mathrm{R}_{\mathrm{u}}+\frac{1}{2} \mathrm{H}_{\mathrm{v}} \mathrm{e}^{\omega} \\
0
\end{array}\right)
$$

The matrix in (11) will be the zero matrix provided the following equations hold:

$$
\begin{aligned}
& \omega_{\mathrm{uv}}+\frac{1}{2} \mathrm{H}^{2} \mathrm{e}^{\omega}-2 \mathrm{RQe}^{-\omega}=0 \\
& \mathrm{H}_{\mathrm{u}}-2 \mathrm{Q}_{\mathrm{v}} \mathrm{e}^{-\omega}, \quad \mathrm{H}_{\mathrm{v}}-2 \mathrm{R}_{\mathrm{u}} \mathrm{e}^{-\omega}
\end{aligned}
$$

These equations will be referred to as the GaussCodazzi equations. Based on the matrices $\mathrm{U}$ and $\mathrm{V}$ given in (9), the equations for the frame (8) can be written down as well:

$$
\begin{aligned}
& \left(\begin{array}{c}
\varphi_{\mathrm{uu}} \\
\varphi_{\mathrm{vu}} \\
\mathrm{N}_{\mathrm{u}}
\end{array}\right)=\mathrm{U}\left(\begin{array}{c}
\varphi_{\mathrm{u}} \\
\varphi_{\mathrm{v}} \\
\mathrm{N}
\end{array}\right)=\left(\begin{array}{c}
\omega_{\mathrm{u}} \varphi_{\mathrm{u}}+\mathrm{QN} \\
\frac{1}{2} \mathrm{H} \mathrm{Ne}^{\omega} \\
-\varphi_{\mathrm{u}} \mathrm{H}-2 \varphi_{\mathrm{v}} \mathrm{Qe}^{-\omega}
\end{array}\right), \\
& \left(\begin{array}{c}
\varphi_{\mathrm{uv}} \\
\varphi_{\mathrm{vv}} \\
\mathrm{N}_{\mathrm{v}}
\end{array}\right)=\mathrm{U}\left(\begin{array}{c}
\varphi_{\mathrm{u}} \\
\varphi_{\mathrm{v}} \\
\mathrm{N}
\end{array}\right)=\left(\begin{array}{c}
\frac{1}{2} \mathrm{HNe}^{\omega} \\
\omega_{\mathrm{v}} \varphi_{\mathrm{v}}+\mathrm{RN} \\
-\varphi_{\mathrm{v}} \mathrm{H}-2 \varphi_{\mathrm{u}} \mathrm{Re}^{-\omega}
\end{array}\right)
\end{aligned}
$$

It will now be shown how this frame can be described within the context of a new algebraic structure and that this new form can be used to determine a two-by-two representation of (12).

Theorem 1: Every nontotally umbilic timelike constant mean curvature surface has a one parameter family of nontrivial isometric deformations preserving the mean curvature.

This is easy to see. On a constant mean curvature timelike surface $\mathrm{M}$, the Gauss-Codazzi equations are invariant under the deformation $\mathrm{Q} \rightarrow \mathrm{Q}_{\lambda}=\lambda \mathrm{Q}$, $\mathrm{R} \rightarrow \mathrm{R}_{\lambda}=\lambda^{-1} \mathrm{R}$. Integrating the deformed Gauss-Codazzi equation, one obtains a one-parameter family of timelike surfaces. This deformation does not effect the induced metric and the mean curvature. Hence all the surfaces are isometric and have the same constant mean curvature.

There is a quaternionic description of surfaces in this space. Let $\varphi: M \rightarrow E_{1}^{3}$ be a timelike surface with moving frame $\left(\varphi_{u}, \varphi_{v}, N\right)$ and define a frame $\Phi$ by means of:

$$
\operatorname{Ad}(\Phi)\left(\mathrm{i}, \mathrm{j}^{\prime}, \mathrm{k}^{\prime}\right)=\left(\mathrm{e}^{-\omega / 2} \varphi_{\mathrm{x}}, \mathrm{e}^{-\omega / 2} \varphi_{\mathrm{y}}, \mathrm{N}\right), \quad \operatorname{det} \Phi=\mathrm{e}^{\omega / 2}
$$

The 3-dimensional Minkowski spacetime $\mathrm{E}_{1}^{3}$ is naturally identified with the imaginary part of the splitquaternion algebra:

$$
\operatorname{Im} H^{\prime}=\left\{\alpha_{1} i+\alpha_{2} j^{\prime}+\alpha_{3} k^{\prime} \mid \alpha_{i} \in \mathbb{R}\right\}
$$

The split-quaternion algebra $\mathrm{H}^{\prime}$ is a real algebra spanned by the basis $\left\{1, i, j^{\prime}, k^{\prime}\right\}$. The multiplication in $\mathrm{H}^{\prime}$ is defined as follows:

$$
\begin{aligned}
& i j^{\prime}=-j i^{\prime}=k^{\prime}, \quad j^{\prime} k^{\prime}=-k^{\prime} j^{\prime}=-i, \quad k^{\prime} i=i k^{\prime}=j^{\prime}, \\
& i^{2}=-1, \quad j^{\prime 2}=k^{\prime 2}=1
\end{aligned}
$$

For a split quaternion $\xi=\xi_{0} 1+\xi_{1} i+\xi_{2} \mathrm{j}^{\prime}+\xi_{3} \mathrm{k}^{\prime}$, the conjugate $\bar{\xi}$ of $\xi$ is defined by $\bar{\xi}=\xi_{0} 1-\xi_{1} \mathrm{i}-\xi_{2} \mathrm{j}^{\prime}-\xi_{3} \mathrm{k}^{\prime}$ and clearly they satisfy $-\xi \bar{\xi}=-\xi_{0}^{2}-\xi_{1}^{2}-\xi_{2}^{2}-\xi_{3}^{2}$. Thus $\mathrm{H}^{\prime}$ can be identified with a semi-Euclidean space: 
$\mathrm{E}_{1}^{4}=\left\{\mathbb{R}^{4}\left(\xi_{0}, \xi_{1}, \xi_{2}, \xi_{3}\right),-\mathrm{d} \xi_{0}^{2}-\mathrm{d} \xi_{1}^{2}-\mathrm{d} \xi_{2}^{2}-\mathrm{d} \xi_{3}^{2}\right\}$

Let $\mathrm{G}=\left\{\xi \in \mathrm{H}^{\prime} \mid \xi \bar{\xi}=1\right\}$ be the multiplication group of the timelike unit split-quaternions. The Lie algebra $g$ of $\mathrm{G}$ is the imaginary part $\mathrm{H}^{\prime}$ :

$$
\mathrm{g}=\operatorname{Im} \mathrm{H}^{\prime}=\left\{\xi_{1} \mathrm{i}+\xi_{2} \mathrm{j}^{\prime}+\xi_{3} \mathrm{k}^{\prime} \mid \xi_{\mathrm{i}} \in \mathbb{R}\right\}
$$

The Lie bracket of $\mathrm{g}$ is simply the commutator of the split-quaternion product and the commutation rules for $g$ are given by $\left[i, j^{\prime}\right]=2 k^{\prime},\left[j^{\prime}, k^{\prime}\right]=-2 i,\left[k^{\prime}, i\right]=2 j^{\prime}$. The Lie algebra $g$ is naturally identified with Minkowski 3space:

$\mathrm{E}_{1}^{3}=\left\{\mathbb{R}^{3}\left(\xi_{1}, \xi_{2}, \xi_{3}\right) \mid-\mathrm{d} \xi_{1}^{2}+\mathrm{d} \xi_{2}^{2}+\mathrm{d} \xi_{3}^{2}\right\}$

as a metric linear space and recall (15) as we require. It is most important here to know that there is a matrix representation for $\mathrm{H}^{\prime}$. The basis elements can be written as:

$1 \leftrightarrow\left(\begin{array}{cc}1 & 0 \\ 0 & 1\end{array}\right), \mathrm{i} \leftrightarrow\left(\begin{array}{cc}0 & -1 \\ 1 & 0\end{array}\right), \mathrm{j}^{\prime} \leftrightarrow\left(\begin{array}{cc}0 & 1 \\ 1 & 0\end{array}\right), \mathrm{k}^{\prime} \leftrightarrow\left(\begin{array}{cc}-1 & 0 \\ 0 & 1\end{array}\right)$

Let the last three matrices in (20) be called $\left\{\tau_{1}, \tau_{2}\right.$, $\left.\tau_{3}\right\}$. Therefore, an arbitrary element of $\mathrm{H}^{\prime}$ takes the form:

$\xi=\left(\begin{array}{cc}\xi_{0}-\xi_{3} & -\xi_{1}+\xi_{2} \\ \xi_{1}-\xi_{2} & \xi_{0}+\xi_{3}\end{array}\right)$

Under the identification (20), the group $G$ of timelike unit split-quaternions corresponds to an algebra $\mathrm{M}_{2} \mathrm{R}$ of all real matrices of degree two. The semi-Euclidean matrix of $\mathrm{H}^{\prime}$ corresponds to the following scalar product:

$$
\langle\mathrm{X}, \mathrm{Y}\rangle=\frac{1}{2}(\operatorname{tr}(\mathrm{XY})-\operatorname{tr}(\mathrm{X}) \operatorname{tr}(\mathrm{Y}))
$$

Now let us take $\Phi \in \mathrm{H}^{\prime}$ to be a matrix valued function which transforms the basis $i, j^{\prime}, k^{\prime}$ into the frame $\varphi_{x}, \varphi_{y}, N$ :

$$
\varphi_{\mathrm{x}}=\mathrm{e}^{\omega / 2} \Phi^{-1} \mathrm{i} \Phi, \quad \varphi_{\mathrm{y}}=\mathrm{e}^{\omega / 2} \Phi^{-1} \mathrm{j}^{\prime} \Phi, \quad \mathrm{N}=\Phi^{-1} \mathrm{k}^{\prime} \Phi
$$
that:

Using the matrix representation (20), it follows

$$
\begin{aligned}
& \varphi_{\mathrm{u}}=\frac{1}{2}\left(\varphi_{\mathrm{x}}+\varphi_{\mathrm{y}}\right)=\mathrm{e}^{\omega / 2} \Phi^{-1}\left(\begin{array}{ll}
0 & 0 \\
1 & 0
\end{array}\right) \Phi \\
& \varphi_{\mathrm{v}}=\frac{1}{2}\left(-\varphi_{\mathrm{x}}+\varphi_{\mathrm{y}}\right)=\mathrm{e}^{\omega / 2} \Phi^{-1}\left(\begin{array}{ll}
0 & 1 \\
0 & 0
\end{array}\right) \Phi
\end{aligned}
$$

It is now possible to obtain a two-by-two matrix representation of (12) by first introducing the following H0-valued pair:

$\Phi_{\mathrm{u}}=\mathrm{U} \Phi$,

$$
\Phi_{\mathrm{v}}=\mathrm{V} \Phi
$$

where $\mathrm{U}$ and $\mathrm{V}$ are defined to be the traceless matrices:

$\mathrm{U}=\left(\begin{array}{ll}\mathrm{U}_{11} & \mathrm{U}_{12} \\ \mathrm{U}_{21} & \mathrm{U}_{22}\end{array}\right), \quad \mathrm{V}=\left(\begin{array}{ll}\mathrm{V}_{11} & \mathrm{~V}_{12} \\ \mathrm{~V}_{21} & \mathrm{~V}_{22}\end{array}\right)$

Explicit forms for the matrix elements of $\mathrm{U}$ and $\mathrm{V}$ can be obtained by enforcing both sides of (13) using the representation (24) and the forms given by (26) for $\mathrm{U}$ and V. Requiring that $\varphi_{\mathrm{uv}}=\varphi_{\mathrm{vu}}$ implies that $\mathrm{U}$ and $\mathrm{V}$ must satisfy:

$\mathrm{U}_{\mathrm{v}}-\mathrm{V}_{\mathrm{u}}+\mathrm{UV}-\mathrm{VU}=0$

To carry out this procedure, begin with $\varphi_{u}$ in (24) and differentiate it with respect to u to obtain:

$$
\begin{gathered}
\varphi_{\mathrm{uu}}=\frac{1}{2} \omega_{\mathrm{u}} \mathrm{e}^{\omega / 2} \Phi^{-1}\left(\begin{array}{ll}
0 & 0 \\
1 & 0
\end{array}\right) \Phi-\mathrm{e}^{\omega / 2} \Phi^{-1} \mathrm{U}\left(\begin{array}{ll}
0 & 0 \\
1 & 0
\end{array}\right) \Phi+ \\
\mathrm{e}^{\omega / 2} \Phi^{-1}\left(\begin{array}{ll}
0 & 0 \\
1 & 0
\end{array}\right) \\
=\mathrm{e}^{\omega / 2} \Phi^{-1}\left(\begin{array}{cc}
-\mathrm{U}_{12} & 0 \\
\frac{1}{2} \omega_{\mathrm{u}}+\mathrm{U}_{11}-\mathrm{U}_{22} & \mathrm{U}_{12}
\end{array}\right) \Phi
\end{gathered}
$$

Using the matrix representation (24) and (23) for $\mathrm{N}$, it is also clear that:

$\omega_{\mathrm{u}} \varphi_{\mathrm{u}}+\mathrm{QN}=\mathrm{e}^{\omega / 2} \Phi^{-1}\left(\begin{array}{cc}-\mathrm{Qe}^{-\omega / 2} & 0 \\ \omega_{\mathrm{u}} & -\mathrm{Qe}^{-\omega / 2}\end{array}\right) \Phi$

The results in (13) imply that (28) and (29) be the same. This serves to fix two of the elements of the matrix $\mathrm{U}$, namely $\mathrm{U}_{11}=\omega_{\mathrm{u}} / 4=-\mathrm{U}_{22}$ and $\mathrm{U}_{12}=\mathrm{Qe}^{-\omega / 2}$. In a similar way, the second derivatives of $\varphi$ with respect to $v$ can be evaluated using (24) and we find that:

$$
\varphi_{\mathrm{vv}}=\mathrm{e}^{\mathrm{\omega} / 2} \Phi^{-1}\left(\begin{array}{cc}
\mathrm{V}_{21} & \frac{1}{2} \omega_{\mathrm{v}}-\mathrm{V}_{11}+\mathrm{V}_{22} \\
0 & -\mathrm{V}_{21}
\end{array}\right) \Phi
$$


$\varphi_{\mathrm{uv}}=\mathrm{e}^{\mathrm{\omega} / 2} \Phi^{-1}\left(\begin{array}{cc}-\mathrm{V}_{12} & 0 \\ \frac{1}{2} \omega_{\mathrm{v}}-\mathrm{V}_{22}+\mathrm{V}_{11} & \mathrm{~V}_{12}\end{array}\right) \Phi$

$\varphi_{\mathrm{vu}}=\mathrm{e}^{\omega / 2} \Phi^{-1}\left(\begin{array}{cc}\mathrm{U}_{21} & \frac{1}{2} \omega_{\mathrm{u}}-\mathrm{U}_{11}+\mathrm{U}_{22} \\ 0 & -\mathrm{U}_{21}\end{array}\right) \Phi$

Using (30-32) with (13), the remaining elements of $\mathrm{U}$ and $\mathrm{V}$ can be calculated just as in the previous case. These results are summarized in the following Theorem.

Theorem 2: Under the isomorphism $\mathrm{Y} \rightarrow \sum_{\alpha=1}^{3} \mathrm{X}_{\alpha} \tau_{\alpha} \rightarrow\left(\mathrm{X}_{1}, \mathrm{X}_{2}, \mathrm{X}_{3}\right)$ in Minkowski three space, the moving frame $\left(\varphi_{\mathrm{u}}, \varphi_{\mathrm{v}}, \mathrm{N}\right)$ of the parameterized surface is described by $\varphi_{\mathrm{u}}, \varphi_{\mathrm{v}}$ given by (24), where $\Phi \in \mathrm{H}^{\prime}$ and the matrices $\mathrm{U}$ and $\mathrm{V}$ in (25) and (27) are given by:

$$
\mathrm{U}=\left(\begin{array}{cc}
\frac{1}{4} \omega_{\mathrm{u}} & \mathrm{Qe}^{-\omega / 2} \\
-\frac{1}{2} \mathrm{He}^{\omega / 2} & -\frac{1}{4} \omega_{\mathrm{u}}
\end{array}\right), \quad \mathrm{V}=\left(\begin{array}{cc}
-\frac{1}{4} \omega_{\mathrm{v}} & \frac{1}{2} \mathrm{He}^{\omega / 2} \\
-\mathrm{Re}^{-\omega / 2} & \frac{1}{2} \omega_{\mathrm{v}}
\end{array}\right)
$$

The quantity $\Phi$, which is regarded as an H0-valued quantity satisfies the pair of Eq. 25 .

To show that (25) is an equivalent form of the Gauss-Codazzi Eq. 12, it is required that the pair in (33) satisfy the condition (27) obtained from the compatibility condition for (25). Calculating this, the result gives the following Theorem.

Theorem 3: System (33) satisfies (27) if and only if the Gauss-Codazzi Eq. 1) are satisfied by the respective functions $\omega, \mathrm{Q}$ and R. Therefore, (25), (33) represent a two-by-two equivalent matrix form of (8) and (10).

As a simple example of a solution to the frame Eq. 25 can be solved explicitly for the vacuum solution $\omega=0$. The matrix $\Phi \in \mathrm{H}^{\prime}$ corresponding to the vacuum solution is given by:

$$
\begin{aligned}
& \Phi=\left(\begin{array}{ll}
\cosh \left(\frac{1}{2}(\mathrm{u}-\mathrm{v})\right) & \sinh \left(\frac{1}{2}(\mathrm{u}-\mathrm{v})\right) \\
\sinh \left(\frac{1}{2}(\mathrm{u}-\mathrm{v})\right) & \cosh \left(\frac{1}{2}(\mathrm{u}-\mathrm{v})\right)
\end{array}\right), \\
& \mathrm{Q}=\frac{1}{2}, \quad \mathrm{R}=1, \quad \mathrm{H}=-1
\end{aligned}
$$

The Gauss-Codazzi Eq. 12 imply that for every simply connected timelike constant mean curvature surface in $E_{1}^{3}$, there exists a timelike extremal surface in $S_{1}^{3}$ which is isometric to the original. Of course, timelike extremal surfaces in $S_{1}^{3}$ can be regarded as simple mathematical models of rigid strings in particle theories as well.

\section{REFERENCES}

Bracken, P. and A.M. Grundland, 1999. On certain classes of solutions of the Weierstrass-Ennper system inducing constant mean curvature surfaces. J. Nonlinear Math. Phys., 6: 294-313.

Bracken, P. and J. Hayes, 2002. On a formulation of certain integrable systems using hyperbolic numbers. Phys. Lett. A., 301: 191-194.

Bracken, P., 2004. Quaternionic representation of the moving frame for surfaces in Euclidean three-space and Lax pair. Int. J. Math. Math. Sci., 15: 755-762.

Bracken, P., A.M. Grundland and L. Martina, 1999. The Weierstrass-Enneper system for constant mean curvature surfaces and the completely integrable sigma model. J. Math. Phys., 40: 3379-3403.

Dorfmeister, J., J. Inoguchi and M. Toda, 2002. Weierstrass type representations of timelike surfaces with constant mean curvature. Integ. Syst. Differ. Geom. Contemp. Math., 308: 77-99.

Inoguchi, J., 1998. Timelike surfaces of constant mean curvature in Minkowski 3-space. Tokyo J. Math., 21: 141-152.

Inoguchi, J., 1999. Darboux transformations on timelike constant mean curvature surfaces. J. Geom. Phys., 32: 57-78.

Konopelchenko, B.G. and I.A. Taimanov, 1996. Constant mean curvature surfaces via an integrable dynamical system. J. Phys. A., 29: 1261-1265.

Weinstein, T., 1996. An introduction to Lorentz surfaces, de Gruyter Exposition in Mathematics, Walter de Gruyter, Berlin, ISBN: 10: 311014333X, pp: 213. 